Ann. Zootech., I966, 15 (2), I7I-I80.

\title{
INTERACTIONS ENTRE GENOTYPE ET MILIEU D'ENGRAISSEMENT CHEZ DES PORCS DE RACE LARGE WHITE, NORMANDE ET DE PIÉTRAIN
}

\author{
L. OLIIVIER \\ avec la collaboration technique de N. GaudiN \\ Station centrale de Génétique animale, \\ Centre national de Recherches zootechniques, 78-Jouy-en-Josas
}

\section{SOMMAIRE}

Dans cette expérience, les descendances de 17 verrats ont été engraissées simultanément en porcherie et en plein air, chaque portée étant divisée au sevrage en deux groupes de 3 porcs placés, au hasard, dans l'un ou l'autre milieu. L'analyse statistique démontre :

- qu'il n'y a d'interaction génotype-milieu pour aucune des mesures de carcasse à l'intérieur des 3 races étudiées ;

- qu'une interaction hautement significative existe en race Normande pour la vitesse de croissance entre 25 et $100 \mathrm{~kg}$;

- que des interactions race-milieu existent, qui sont imputables aux mauvaises conditions sanitaires de la porcherie au cours des deux dernières années de l'expérience.

\section{INTRODUCTION}

Pour la sélection du porc, on emploie des stations de grande capacité dans lesquelles on s'efforce d'uniformiser les conditions de l'engraissement; les porcheries utilisées à cet effet sont, le plus souvent, climatisées et les porcs y sont logés en cases individuelles. Il est donc important de savoir si le classement des verrats obtenu dans le milieu bien particulier de ces stations reste le même dans les conditions de la pratique courante, ou, en d'autres termes, s'il y a interaction entre le génotype de l'animal et le milieu dans lequel il vit. 
Une grande diversité apparaît dans les schémas expérimentaux des expériences d'interaction génotype-milieu, aussi bien en ce qui concerne les génotypes étudiés que les conditions de milieu, les caractères mesurés et les effectifs mis en œuvre. Comme le font remarquer HALE et COEX (1962) dans une revue bibliographique très détaillée, presque toutes ces expériences concernent des races ou des lignées différentes et des nilieux représentés par un mode d'alimentation restreinte en comparaison avec une alimentation à volonté. Il en ressort que des interactions entre le facteur race et le facteur milieu s'observent fréquemment. Par contre, à l'intérieur d'une race, les études d'interaction entre le facteur "verrat " et les conditions d'élevage sont plus rares et les résultats contradictoires.

L'expérience que nous présentons ici a été réalisée au C. N. R. Z. de I956 à I959 ( ${ }^{(1)}$; elle avait pour but de savoir si des conditions d'élevage aussi différentes que le plein air avec alimentation en groupe d'une part et la porcherie climatisée avec alimentation individuelle d'autre part peuvent influencer des comparaisons entre races, ou modifier le classement des verrats dans une race sur la valeur de leur descendance.

\section{PIAN DE L'EXXṔRIENCE}

\section{$\mathbf{I}^{\circ}$ Les génotypes}

Le troupeau expérimental de la Station de Recherches sur l'Élevage a fourni les 317 animaux utilisés dans cette étude, répartis en trois races, Large White, Normande et race de Piétrain, et descendant de $\mathrm{I} 7$ verrats, neuf en race Large White, quatre en race Normande et quatre en race de Piétrain.

\section{$2^{\mathrm{O}}$ Les milieux}

Au sevrage, chaque portée a été divisée en deux groupes de 3 porcelets (le plus souvent deux mâles castrés et une femelle ou l'inverse) attribués au hasard à l'un des deux milieux suivants :

a) une porcherie de mise à l'épreuve des verrats sur leur descendance située à Jouy-en-Josas. Cette porcherie était chauffée en hiver et les porcs y étaient placés en loges individuelles avec alimentation en trois repas par jour selon l'appétit de l'animal.

b) une instailation de plein air, située sur le domaine de La Ilaizerie, à Vaux-sur-Aure (Calvados) et constituée d'enclos grillagés avec abris sommaires en ballots de paille. Chaque enclos recevait un groupe de trois porcs de la même portée, soumis à une alimentation collective distribuée en deux repas par jour suivant l'appétit du groupe.

\section{$3^{\circ}$ Les caractères étudiés}

Les caractères suivants ont été pris en considération :

a) l'âge de l'animal en fin d'engraissement, c'est-à-dire, vers $100 \mathrm{~kg}$;

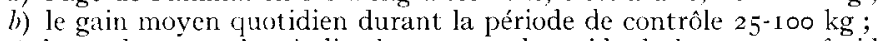

c) le rendement, c'est-a-dire le rapport du poids de la carcasse froide, sans tête, au poids vif avant l'abittage ;

d) la proportion de morccaux nobles (jambon et longe) dans la carcasse ;

e) la proportion de morceaux gras (bardière et panne) dans la carcasse ;

f) l'épaisseur de lard, moyenne de deux mesures prises l'une à la dernière vertèbre dorsale, l'autre à la dernière vertèbre lombaire ;

g) la longueur de la carcasse, mesurée de l'atlas au pubis, la carcasse étant suspendue.

Les abat tages et les mesures sur carcasse ont été réalisés dans les abattoirs du C. X. R. Z.

(1) A l'initiative de R. Févrlkr, alors directeur de la Station de Recherches sur l'Élevage. 


\section{MÉTHODES D'ANALYSE}

\section{A -- Analyse préliminaire du gain moyen quotidien}

Le gain moyen dépend, dans une certaine mesure, du poids atteint au début de la période de contrôle. Or, les poids en début d'engraissement ont varié différemment selon les conditions de milieu. En porcheric, avec engraissement en loges individuelles, il est aisé de maintenir ces poids dans des limites étroites. En fait, dans cette étude, 12 porcs seulement sur les 158 étudiés en porcherie ont des poids inféricurs à $24 \mathrm{~kg}$ ou supérieurs à $27 \mathrm{~kg}$. Par contre, dans les conditions d'une alimentation en groupe, les poids initiaux sont nécessairement plus variables, ce qui oblige à corriger le gain moyen observé en fonction du poids initial. La linéarité des régressions du gain moyen sur ce poids initial a été testée dans les deux milieux. Ces régressions peuvent être considérées comme linéaires $\left(F=I, 37\right.$ pour la porcherie, avec 6 et $15^{6}$ degrés de liberté, $F=1,52$ pour le plein air avec I4 et I 5 I degrés de liberté). Le gain moyen augmente en moyenne de 1,3 $\mathrm{g}$ par jour en porcherie et de $5,5 \mathrm{~g}$ par jour en plein air pour une augmentation de $\mathrm{I} \mathrm{kg}$ du poids en début d'engraissement. Toutes les données ont été corrigées en fonction de ces régressions.

D'autre part, plusieurs études ayant montré que la variance du gain moyen en alimentation individuelle est nettement inférieure à celle observée en alimentation collective, les variances intragroupe, dans chaque milieu d'engraissement, ont été estimées, après correction pour le poids initial, et comparées entre elles par un test F. Ces variances sont respectivement égales à 3685 en porcherie et 3437 en plein air et la différence ri'est pas significative.

\section{B - Regroupement des données}

Par suite de la durée de l'expérience, des facteurs de variation imprévus sont intervenus. D'abord, les conditions d'élevage entre la naissance et le sevrage n'ont pas été uniformes, les portées étant élevées, soit en plein air, soit en porcherie. Ensuite, un état sanitaire défectueux a entraîné en $195^{8}$ et 1959 une chute importante de la vitesse de croissance dans la porcherie, comme en témoignent les moyennes suivantes établies sur des animaux de race Large W'hite en provenance d'élevages inscrits:

\begin{tabular}{cccccc} 
& \multicolumn{5}{c}{ Année d'abattage } \\
Gain moyen quotidien $(\mathrm{g})$ de 25 à $100 \mathrm{~kg} \ldots \ldots$ & 59.56 & $19.5 \%$ & 19.58 & 19.59 \\
& & 512 & 548 & $57 \mathrm{I}$
\end{tabular}

Pour tenir compte de ces variations, on a constitué des ensembles d'animaux de même race, nés au même endroit et contrôlés au cours de la même période, comme suit :

Ènsemble I : animaux de race Large White, nés en porcherie, abattus en 1956-1957.

Ensemble 2: animaux de race Large White, nés en plein air, abattus en $195^{8-1959 .}$

Ensemble 3 : animaux de race Nornande, nés en plein air, abattus en 1956-1957.

Ensemble 4: animaux de race de P'iétrain, nés en porcherie, abattus en 1958-59.

\section{$\mathrm{C}-$ Test des interactions}

L'analyse statistique que nous allons décrire a d'abord été appliquée séparément aux quatre ensembles définis ci-dessus, les facteurs de variation considérés étant le "verrat ", le " sexe "et le " lieu ". Dans un deuxième stade, les ensembles contemporains, I et 3 d'une part, 2 et 4 d'autre part, ont été fusionnés, les facteurs de variation considérés étant alors la "race "le " sexe " et le "lieu ". Il est à remarquer que le facteur "race " est ici confondu avec les conditions d'élevage entre la naissance et le sevrage.

\section{Caractéristiques d'engraissement.}

Pour l'âge en fin d'engraissement et le gain moyen quotidien, le facteur "sexe " n'a pas été considéré. Les différences entre mâles et femelles sont généralement faibles, et dans les échantillons étudiés ici elles ne sont en aucun cas significatives. Les effets attachés à chacun des niveaux des facteurs 
"verrat " (ou " race ") et " lieu " ont été estimés par la méthode des moindres carrés, en supposant l'absence d'interaction. La variation non expliquée par cet ajustement a alors été comparée à la variation résiduelle, ce qui constitue un test de l'interaction " verrat " (ou " race ") $x$ " lieu ".

2. Caractéristiques de carcasse.

Pour l'ensemble des mesures sur carcasse, il n'est pas possible de négliger le facteur " sexe". Les effets attachés aux facteurs " verrat " (ou " race "), "sexe " et " lieu " ont été estimés, comme précédemment, en supposant l'absence de toute interaction et la variation non expliquée par cet ajustement a été comparée à la variation résiduelle, ce qui constitue un test global des quatre interactions. Les interactions de premier ordre ont été soumises à un test selon le même principe, en faisant abstraction du troisième facteur. Ce test n'est pas rigoureux car il suppose que la répartition des effectifs selon les niveaux du facteur négligé n'influe pas sur les comparaisons.

Tous les calculs qui viennent d'être décrits ont été réalisés mécanographiquement à la Station centrale de Génétique animale.

\section{RÉSULTATS}

I.es moyennes de chaque caractère étudié sont présentées dans le tableau I.

TABLEAU I

Résultats moyens par race, période et lieu d'engraissement

\begin{tabular}{|c|c|c|c|c|c|c|c|c|}
\hline $\begin{array}{l}\text { Ensemble . . . . . . } \\
\text { Race............ } \\
\text { Années . . . . . . . }\end{array}$ & \multicolumn{2}{|c|}{$\begin{array}{c}\text { (1) } \\
\text { Large White } \\
1956-57\end{array}$} & \multicolumn{2}{|c|}{$\begin{array}{c}(2) \\
\text { Large White } \\
1958-59\end{array}$} & \multicolumn{2}{|c|}{$\begin{array}{c}(3) \\
\text { Normande } \\
1956-57\end{array}$} & \multicolumn{2}{|c|}{$\begin{array}{l}(4) \\
\text { Piétraitr } \\
1958-59\end{array}$} \\
\hline Lieu de naissance.... & \multicolumn{2}{|c|}{ Porcherie } & \multicolumn{2}{|c|}{ I'lein air } & \multicolumn{2}{|c|}{ Plein air } & \multicolumn{2}{|c|}{ Porcherie } \\
\hline Lieu d'engraissement . i & cherie & lein a & Porcheri & lein air & Porcherie & Plein air & Porcherie & Plein air \\
\hline Effectif total ....... & 38 & 43 & 57 & 51 & 26 & 96 & 37 & $3 ! 4$ \\
\hline Hâles castrés . . . . . . . & 27 & 95 & 35 & 28 & 19 & 15 & 15 & 17 \\
\hline $\begin{array}{l}\text { Hemelles } \ldots . \cdots \cdots \\
\text { Age en fin d'engrais- }\end{array}$ & 11 & 18 & 24 & 23 & 7 & 11 & 22 & 22 \\
\hline $\begin{array}{l}\text { sement (jours) ..... } \\
\text { Gain moyen quotidien }\end{array}$ & 225 & 219 & $20^{\prime} 0$ & 213 & 222 & $\because 06$ & 271 & 257 \\
\hline (g) de 25 a $100 \mathrm{~kg}$. & 625 & 620 & 523 & 596 & 573 & 584 & 491 & $51^{\prime}$ \\
\hline Rendement $(\%) \ldots .$. & 71,91 & 74,33 & 72,25 & $7: 3,82$ & 70,53 & 72,85 & $7 \div, 29$ & 74,62 \\
\hline Jambon + Longe $(\%)$ & 50,10 & 48.85 & 50,53 & $\{9,4 \div 2$ & $51, \underline{97}$ & 50,11 & $5^{\prime}, 50$ & 55,03 \\
\hline Bardière \& Panne $(\%)$ & 19,70 & 19,92 & 19,95 & 21,15 & 17,81 & 17,30 & 15,58 & 15,33 \\
\hline Epaisseur de lard (mm) & 27,61 & 28,75 & 29,26 & 30,31 & $2 ', 23$ & 25,83 & 23,44 & 22,40 \\
\hline Iongueur $(\mathrm{cm}) \ldots \ldots$. & 96,19 & $97, \geq 3$ & 94,98 & 96,33 & 95,15 & 96,37 & 90,22 & $91, \mathbf{1} 0$ \\
\hline
\end{tabular}

\section{Io Caractéristiques d'engraissement}

Les résultats des tests statistiques sont groupés dans le tableau 2 . Des interactions significatives entre "verrat " et " lieu " existent pour la période I956-I957 aussi bien en race Large White qu'en race Normande. Cependant, dans la première race, l'interaction ne concerne que l'âge en fin d'engraissement, le gain moyen n'étant pas influencé par le facteur " lieu ". L'interaction se manifeste donc vraisemblable- 
ment avant le début de la période de contrôle. Elle pourrait s'expliquer par une influence du verrat sur la faculté d'adaptation de ses descendants aux conditions de la porcherie. Car, entre le sevrage et le début du contrôle il peut s'écouler un laps de temps supérieur à un mois. Ainsi, deux des verrats Large White étudiés ont

TABLEAU 2

Analyses de variance des caractéristiques d'engraissement

\begin{tabular}{|c|c|c|c|c|}
\hline \multirow[b]{2}{*}{ Emsemble } & \multirow[b]{2}{*}{ Cause de variation } & \multirow{2}{*}{$\begin{array}{c}\text { Degrés } \\
\text { de } \\
\text { liberté }\end{array}$} & \multicolumn{2}{|c|}{ Carrés moyens } \\
\hline & & & $\begin{array}{c}\text { Age en fin d'engrais- } \\
\text { sement } \\
\text { (jour) }\end{array}$ & $\begin{array}{l}\text { Gain moyen quotidien } \\
\text { (g) de } 25 \text { à } 100 \mathrm{k}\end{array}$ \\
\hline $\begin{array}{c}\text { (1) } \\
\text { Race Large White } \\
195 \hat{6}-1957\end{array}$ & $\begin{array}{l}\begin{array}{l}\text { Interaction Verrat } \times \text { Lieu.... } \\
\text { Verrat } \ldots \ldots \ldots \ldots \ldots \ldots \ldots\end{array} \\
\text { |Lieu } \ldots \ldots \ldots \ldots \ldots \ldots \ldots \ldots \ldots\end{array}$ & $\begin{array}{r}5 \\
5 \\
1 \\
69\end{array}$ & $\begin{array}{l}576 * \\
- \\
-11\end{array}$ & $\begin{array}{r}6457 \\
1805 \\
526 \\
4125\end{array}$ \\
\hline $\begin{array}{c}(2) \\
\text { Race Large Wihte } \\
1958-1959\end{array}$ & $\begin{array}{l}\text { Interaction Verrat } \times \text { Lieu. } \ldots \\
\text { Verrat } \ldots \ldots \ldots \ldots \ldots \ldots \ldots \\
\begin{array}{|l}\text { Lieu } \ldots \ldots \ldots \ldots \\
\text { Résiduelle } \ldots \ldots \ldots \ldots \ldots\end{array}\end{array}$ & $\begin{array}{r}2 \\
2 \\
1 \\
102\end{array}$ & $\begin{array}{rl}1 & 296 \\
1 & 982^{*} \\
18 & 782^{* *} \\
& 535\end{array}$ & $\begin{aligned} 5327 \\
973 \\
141737^{* *} \\
\quad 1565\end{aligned}$ \\
\hline $\begin{array}{c}\text { Race Normande } \\
1956-1957\end{array}$ & $\begin{array}{l}\text { Interaction Verrat } \times \text { Lieı.... } \\
\text { Verrat } \ldots \ldots \ldots \ldots \ldots \ldots \ldots \ldots \ldots \ldots \\
\text { Lieu } \ldots \ldots \ldots \ldots \ldots \ldots \ldots \ldots \ldots \\
\text { Résiduelle } \ldots \ldots \ldots \ldots \ldots \ldots\end{array}$ & $\begin{array}{r}3 \\
3 \\
1 \\
4\end{array}$ & $\frac{1198^{*}}{-}$ & $\begin{array}{c}\text { 1' } 680^{* * *} \\
- \\
30\end{array}$ \\
\hline $\begin{array}{l}(1) \\
\text { Race de Pietrain } \\
1958-1959\end{array}$ & 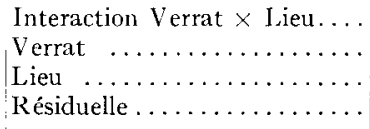 & $\begin{array}{r}3 \\
3 \\
1 \\
68\end{array}$ & $\begin{array}{rl} & 238 \\
1 & 72 / \\
4 & 106^{*} \\
& 713\end{array}$ & $\begin{array}{l}3105 \\
5978 \\
6555 \\
4218\end{array}$ \\
\hline $\begin{array}{l}\text { (1) et (3) } \\
1956-1957\end{array}$ & $\begin{array}{l}\text { Interaction Race } \times \text { Lieı } \ldots \ldots \\
\text { Race } \ldots \ldots \ldots \ldots \ldots \ldots \ldots \ldots \\
\text { Lieu } \ldots \ldots \ldots \ldots \ldots \ldots \ldots \ldots \\
\text { Résiduelle } \ldots \ldots \ldots \ldots \ldots\end{array}$ & $\begin{array}{r}1 \\
1 \\
1 \\
129\end{array}$ & $\begin{array}{ll} & 975 \\
2 & 157^{*} \\
3 & 186^{* *} \\
& 338\end{array}$ & $\begin{array}{c}2011 \\
60747^{* *} \\
73 \\
4453\end{array}$ \\
\hline $\begin{array}{l}(2) \text { et }(1) \\
1958-1950\end{array}$ & $\begin{array}{l}\text { Interaction Race } \times \text { Lieu } \ldots \\
\text { Race } \ldots \ldots \ldots \ldots \ldots \ldots \ldots \ldots \ldots \\
\text { Lieu } \ldots \ldots \ldots \ldots \ldots \ldots \ldots \ldots \ldots \\
\text { Résiduelle } \ldots \ldots \ldots \ldots \ldots \ldots\end{array}$ & $\begin{array}{r}1 \\
1 \\
1 \\
180\end{array}$ & $\begin{array}{c}1976 \\
62409^{* *} \\
21351^{* *} \\
611\end{array}$ & $\begin{array}{c}31191^{* *} \\
\cdots \\
- \\
113\end{array}$ \\
\hline
\end{tabular}

* Test $\mathrm{F}$ significatif au seuil de $5 \%$.

** Test $\mathrm{F}$ significatif au seuil de $1 \%$.

donné des descendants ayant sensiblement le même gain moyen en porcherie mais dont les âges à 1'abattage diffèrent en moyenne de $I_{5}$ jours. En ce qui concerne la race Normande, l'interaction verrat $\times$ lieu est hautement significative pour le gain moyen et suffit à expliquer l'interaction également observée pour l'âge en fin d'engraissement. 
Parmi les interactions " race " $x$ " lieu ", une seule se montre significative, il s'agit du gain moyen pour la période I958-r959. La race de Piétrain a réagi différemment de la race Large White aux conditions défavorables qui régnaient en porcherie durant cette période, la chute de gain moyen étant de $20 \mathrm{~g}$ par jour pour la première race contre 73 pour la deuxième.

Les conclusions suivantes découlent de la comparaison plein air-porcherie en I956-I957, période durant laquelle les conditions de porcherie peuvent être considérées comme normales. L'âge en fin d'engraissement est significativement accru en

TABLEAU 3

Analyses de variance des caractéristiques de carcasse

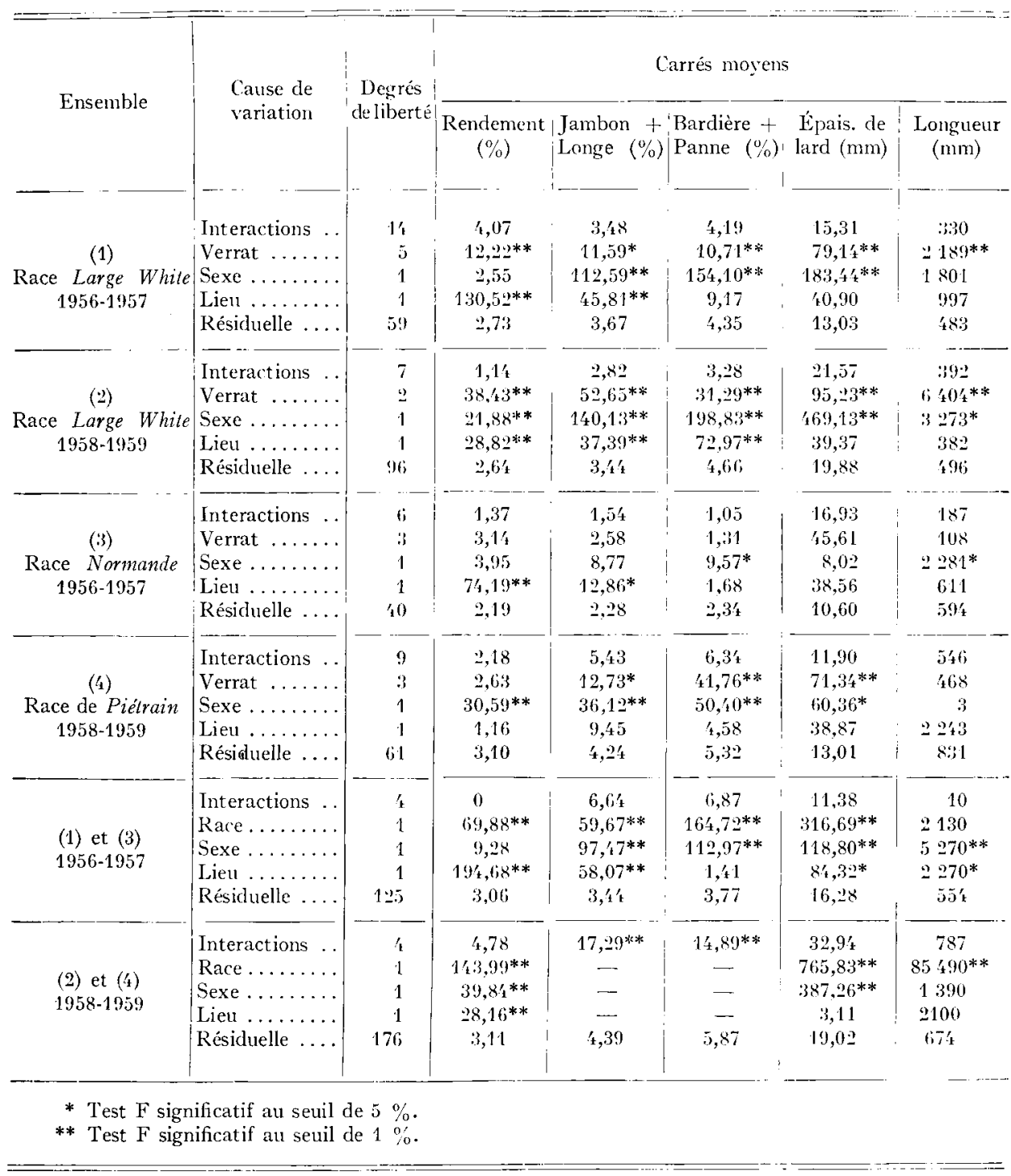


porcherie, mais la croissance entre 25 et Ioo $\mathrm{kg}$ est la même dans les deux milieux, ce qui revient à dire que le porcelet s'adapte plus rapidement à son milieu d'engraissement quant il est placé dans des conditions de plein air que lorsqu'il est mis en porcherie, qu'il ait été jusqu'alors élevé en plein air ou en porcherie.

\section{$2^{\circ}$ Caractéristiques de carcasse}

Les résultats des tests statistiques sont groupés dans le tableau 3. En ce qui concerne les analyses intra-race, les carrés moyens attribuables aux interactions dépassent rarement la variance résiduelle. Par contre, les analyses intra-période révèlent des interactions hautement significatives pour les pourcentages de morceaux nobles et de morceaux gras dans la période I958-I959. Comme indiqué précédemment, l'analyse statistique a été poursuivie en négligeant successivement chacun des facteurs de variation de manière à tester les interactions de premier ordre. Seules les interactions " race " $x$ " lie11 " se sont montrées significatives pour ces deux caractères. Comme le montre le tableau I, en race Large White les mâles sont proportionnellement plus nombreux en plein air qu'en porcherie, alors qu'en race de Piétrain la répartition en mâles et femelles est la même dans les deux lieux d'engraissement. Cependant l'interaction observée est trop importante pour s'expliquer entièrement de cette manière. L,es tests statistiques du tableau 3 démontrent d'ailleurs que l'influence du lieu est hautement significative en race Large White, alors qu'elle ne l'est pas en race de Piétrain.

Parmi les autres interactions de premier ordre qui ont été testées, deux seulement sont significatives. Il s'agit des interactions " race " $x$ " sexe " pour les pourcentages de morceaux nobles et de morceaux gras dans la période I956-1957. La signification de ces interactions est douteuse car il est difficile de dissocier la part due à l'inégalité des effectifs dans les deux lieux et celle due à une réelle interaction. Il est, malgré tout, probable que celle-ci existe car le tableau 3 montre que les différences entre mâles et femelles atteignent des seuils de signification plus élevés en race Large White qu'en race Normande pour ces deux caractères.

La comparaison plein air-porcherie sur la période normale I956-I957 fait ressortir un rendement et une longueur supérieurs en plein air, mais, par contre, en porcherie l'épaisseur du lard est plus faible et la teneur de la carcasse en morceaux nobles est plus élevée qu'en plein air.

\section{DISCUSSION}

L'analyse statistique des résultats a été compliquée par l'inégale répartition des effectifs selon les niveaux des facteurs de variation étudiés et par la longue durée de l'expérience, source d'importantes variations dans les conditions de porcherie. De plus, la comparaison porte sur deux milieux qui diffèrent à la fois par l'habitat, le mode de vie et le mode de distribution de l'aliment, ce qui rend l'interprétation difficile.

Cependant, on ne peut douter de la réalité des interactions mises en évidence par notre méthode d'analyse, à part peut-être l'interaction " race $\times$ sexe » observée sur les pourcentages de morceaux nobles et de morceaux gras en I956-r957. Une telle interaction n'a jamais été signalée, à notre connaissance, et tous les résultats 
des comparaisons entre mâles et femelles aboutissent à la conclusion que les femelles donnent des carcasses moins adipeuses et plus riches en viande que les mâles castrés. Il est cependant possible que ces différences soient plus ou moins accentuées selon les races.

En ce qui concerne les interactions génotype-milieu, examinons d'abord celles observées à l'intérieur de chaque race.

Elles ne concernent que les caractéristiques d'engraissement, l'âge en fin d'engraissement pour la race Large White, le gain moyen quotidien en race Normande. Dans le premier cas, c'est la faculté d'adaptation du porcelet aux conditions de porcherie qui est en cause, sans qu'il soit possible d'incriminer l'environnement général (lumière, température, humidité, etc.) ou le fait de placer l'animal en loge individuelle. Dans le cas de la race Normande, l'interaction concerne la croissance entre 25 et Ioo $\mathrm{kg}$ et n'est peut être pas étrangère au fait que cette race a été élevée et sélectionnée depuis de longues années dans des conditions de plein air. L/es deux expériences qui se rapprochent le plus de celle que nous venons de décrire sont celles de Kristjansson (I957) et de OntveDt et coll. (I962), puisqu'elles comparent toutes les deux des conditions d'élevage en plein air et en porcherie. Leurs résultats sont à l'opposé des nôtres, car aucune interaction n'est significative pour la vitesse de croissance et quelques-unes le sont pour des critères de carcasse (surface de la noix de côtelette, rendement en carcasse, épaisseur du lard sur la carcasse). Cependant, ces auteurs reconnaissent que leur matériel expérimental est particulièrement limité en ce qui concerne le choix des génotypes. Sur l'ensemble des expériences de ce type, il apparait, comme le fait remarquer KING (I963), que les interactions sont plus fréquentes pour les caractères d'engraissement que pour les mesures de la carcasse. Nos résultats accentuent cette tendance.

Quant aux interactions race $x$ milieu, elles ne se manifestent que pour la période I958-I959 et correspondent à un milieu "porcherie " particulièrement défavorable au point de vue sanitaire. La vitesse de croissance en race Large White chute fortement dans de telles conditions, alors qu'en race de Piétrain la baisse est faible et d'ailleurs non significative. Il semble donc que cette dernière race ait présenté une meilleure résistance aux conditions sanitaires défavorables de porcherie. Ceci confirme l'observation de KNOERTZER (I96I), qui note que les porcs de Piétrain sont peu sæjets à la toux même quand les conditions sanitaires sont défectueuses, comme c'était le cas en I958 et I959. Il convient cependant de remarquer ici que la comparaison des deux races n'est pas absolument rigoureuse, parce que les conditions d'élevage entre la naissance et le sevrage n'étaient pas les mêmes : au moment du sevrage, les porcs Large White passaient du plein air à la porcherie, alors que les porcs de Piétrain changeaient seulement de porcherie, ce qui pouvait rendre plus rapide leur adaptation aux conditions de l'engraissement.

\section{CONCLUSION}

Pour la sélection, quelles conclusions peut-on tirer de cette expérience conçue il y a déjà quelques années à une époque où l'alternative plein air-porcherie avait une certaine importance? Il semble qu'actuellement l'engraissement en porcherie 
soit devenu la règle quasi générale ou soit en voie de le devenir. Il reste cependant la question de savoir si l'alimentation individuelle donne des résultats transposables en alimentation collective. La réponse est affirmative pour les critères de carcasse d'après les résultats qui viennent d'être rapportés. Pour la vitesse de croissance la possibilité d'une interaction génotype-milieu existe mais il n'est pas prouvé que la cause en soit réellement l'alimentation individuelle. Rien ne semble donc justifier l'abandon de ce mode d'alimentation dans les stations de mise à l'épreuve des verrats sur leur descendance.

Par ailleurs nos résultats confirment la difficulté de réaliser des comparaisons de race dont les résultats puissent être généralisés, en ce qui concerne les caractéristiques d'engraissement. Celles-ci dépendent en effet pour une part, surtout si le milieu n'est pas parfaitement sain, de la résistance des animaux à diverses infections et nos résultats suggèrent que des différences raciales peuvent exister pour ce caractère, hypothèse qui mériterait d'être vérifiée car ses conséquences pratiques seraient importantes.

Reçu pour publication en mai 1966.

\section{SUMMARY}

INTERACTIONS BETWEEN GENOTYPE AND FATTENING ENVIRONMENT IN " LARGE WHITE ", " NORMAND " AND " PIÉTRAIN " BREEISS OF PIGS

Seventeen boars, belonging to one of the three breeds, Large White, Normand and Pietrain, were simultaneously progeny-tested in a fattening house under individual feeding and in the open air under group feeding, from 1956 to 1959 .

A statistical analysis of the data shows that :

- there is no interaction between genotype and environment within breed for carcass measurements and for the proportions of lean cuts and fat cuts in the carcass;

- there is a highly significant genotype-environment interaction in the Wormand breed for growth rate between 25 and $100 \mathrm{~kg}$ liveweight ;

- breed-environment interactions exist, which are partly due to unfavourable health conditions in the fattening house during the last two years of the experiment.

\section{REMERCIEMENTS}

L'auteur tient à exprimer sa gratitude à M. R. FÉvrier et E. KNOERTzEr, qui ont conduit catte expérience et bien voulu lui confier l'analyse des résultats.

\section{RÉFÉRENCES BIBLIOGRAPHIQUES}

Hate R. W. Coer W. E., rg63. Genotype-environment interaction in a herd of bacon pigs. Agric. Sci., 61, 8 I-88.

Jonsson P., I959. Investigations on group feeding versus individual feeding and on the interaction between genotype and environment in pigs. Acla Agric. Scand., 9, 204-228.

KING J. W. B., I963. A genotypa-environment interaction experiment with bacon pigs. Anim. Prod., 5, $283^{-2}-88$. 
Knoertzer E., 196r. Quelques données biométriques sur le porc de Piétrain. Bull. Tech. Inform. Ingrs Serv. agric., 164, 3-37.

KRISTJANSSON F. K., 1957. Observations on genotype-environment interaction in swine. Canad. J. anim. Sci., 3\%, I79-184.

LUCAS I. A. M., CALDER A. F. C., I956. The response of different types of pigs to varying levels of feeding from weaning to bacon weight, with particular reference to carcass quality. J. agric. Sci., 47, $287-318$.

Omtvedt I. T., Whatley J. A., Whiteman J. V., Morrison R. D., ig62. Genotype-environment interaction in feedlot performance and carcass traits in swine. J. anim. Sci., 21, 4I-48. 